\title{
Evaluación del Aspergillus lateral flow device para el diagnóstico de aspergilosis invasora, experiencia en un hospital universitario
}

\author{
Ignacio Delama', Paulette Legarraga², Tamara González³, Patricia García y Ricardo Rabagliati'
}

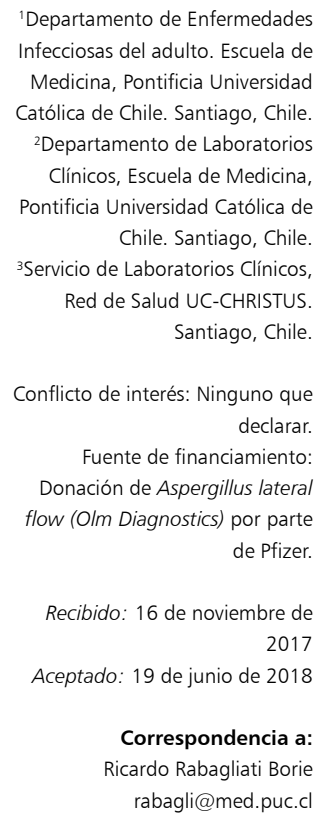

'Departamento de Enfermedades Infecciosas del adulto. Escuela de Medicina, Pontificia Universidad Católica de Chile. Santiago, Chile. ${ }^{2}$ Departamento de Laboratorios Clínicos, Escuela de Medicina, Pontificia Universidad Católica de Chile. Santiago, Chile. ${ }^{3}$ Servicio de Laboratorios Clínicos, Red de Salud UC-CHRISTUS. Santiago, Chile.

Conflicto de interés: Ninguno que declarar.

Fuente de financiamiento: Donación de Aspergillus lateral flow (Olm Diagnostics) por parte de Pfizer.

Recibido: 16 de noviembre de 2017 Aceptado: 19 de junio de 2018

Correspondencia a: Ricardo Rabagliati Borie rabagli@med.puc.cl

\section{Introducción}

L a incidencia de enfermedad fúngica invasora (EFI) ha aumentado en los últimos años ${ }^{1}$. Estudios internacionales muestran una incidencia de hasta $13 \%$ de aspergilosis invasora ( $\mathrm{AI}$ ) en pacientes de riesgo ${ }^{2}$. En nuestro medio, en un estudio prospectivo de etiología de neutropenia febril en pacientes adultos portadores de leucemia aguda y linfoma, EFI fue el responsable de 20\% de estos episodios, siendo AI la etiología predominante ${ }^{3}$. En general, en la mayoría de las series se describe que Aspergillus fumigatus es la especie más frecuentemente identificada ${ }^{4} \mathrm{y}$ entre los factores de riesgo más comunes destacan la presencia de neutropenia profunda y prolongada, tanto en pacientes en quimioterapia por patología maligna hematológica como receptores de trasplantes de precursores hematopoyéticos y el uso prolongado de corticosteroides o inmunosupresores en receptores de trasplante de órganos sólidos o por enfermedad inflamatoria. A pesar de los avances en el diagnóstico y la terapia antifúngica, la mortalidad asociada sigue siendo elevada ${ }^{1}$.

Como en otras EFI, el diagnóstico de AI es difícil, requiriendo evidencia histológica de infiltración de tejidos o cultivo positivo para Aspergillus desde un sitio estéril ${ }^{5}$, resultando clínicamente desafiante obtener muestras de tejido por biopsia en pacientes habitualmente trombocitopénicos. Tanto la visualización directa del hongo como el cultivo presentan una sensibilidad menor a $50 \%$, y si bien las técnicas de biología molecular ya están estandarizadas $^{6}$, aún no están incluidas como criterio diagnóstico según el consenso vigente de EORTC/MSG ${ }^{5,7}$ y requieren de laboratorios de mayor complejidad, dificultando su implementación de manera rutinaria. En este contexto, los marcadores serológicos han surgido como una herramienta de gran utilidad, en especial la determinación de galactomanano (GM) en suero y/o lavado bronco alveolar (LBA), que ha sido incorporada en los criterios diagnósticos de AI de EORTC/MSG ${ }^{5}$. La sensibilidad del GM varía entre 58 y $64 \%{ }^{8}$, requiere de personal entrenado, es de alto costo y tiene tiempos de entrega de resultados variables, de varias horas a días. Además, en pacientes en que se vigila la emergencia precoz de infección por Aspergillus spp., se sugiere su determinación dos a tres veces por semana, lo que implica altos costos del proceso de vigilancia.

Durante el año 2008 se describió el desarrollo de una inmunocromatografía específica para la detección de antígenos liberados durante el crecimiento activo de Aspergillus conocido como Aspergillus lateral flow device $(\text { LFD })^{9}$ mediante el uso de un anticuerpo monoclonal 
Mab JF5 del tipo IgG, específico para una glicoproteína de $40 \mathrm{kDa}$ secretada durante el crecimiento activo de Aspergillus. Este test puede realizarse en muestras de suero o LBA y su lectura se efectúa a los $5 \mathrm{~min}$.

El objetivo de este trabajo fue evaluar el rendimiento diagnóstico de LFD en muestras de suero y/o LBA de pacientes adultos que habían sido sometidos a proceso diagnóstico por sospecha de AI.

\section{Material y Métodos}

\section{Muestras y pacientes}

Estudio de corte transversal, en el que se identificaron muestras de suero y LBA, congeladas a $-20^{\circ} \mathrm{C}$, que habían sido procesadas para la determinación de GM entre los años 2012 y 2015 en el laboratorio de microbiología de la Red de Salud UC-CHRISTUS, correspondientes a pacientes mayores de 15 años internados en el Hospital Clínico de la misma Red, a quienes su médico tratante solicitara el examen por vigilancia, sospecha o seguimiento de AI. Se procedió a revisar las fichas clínicas de los casos, se registraron las variables demográficas, enfermedad de base, estudio imagenológico, resultados de GM, así como los resultados de estudio microbiológico e histológico en la medida que estaban disponibles. Los casos fueron clasificados como episodios de AI probada, probable, posible o ausencia de ésta, según criterios EORTC/MSG ${ }^{5}$.

\section{Aspergillus lateral flow device}

A las muestras seleccionadas se les realizó el test de LFD según instrucciones del fabricante (OLM Diagnostics $\left.{ }^{\circledR}\right)$. En breve, en el caso de muestras de suero, $50 \mu 1$ de muestra fueron calentadas a $50^{\circ} \mathrm{C}$ con $100 \mu \mathrm{L}$ de tampón para luego ser centrifugadas a $14.000 \mathrm{~g}$ durante cinco minutos. Cien $\mu \mathrm{L}$ del sobrenadante fueron depositados en la tira inmunocromatográfica la que fue leída a los cinco minutos (Figura 1). En el caso de muestras de LBA, las muestras fueron depositadas directamente en la tira. Cada muestra fue leída por tres observadores independientes.

\section{Galactomanano}

Las muestras seleccionadas habían sido procesadas en el laboratorio para la detección de GM (Platelia ${ }^{\mathrm{TM}}$ Aspergillus, Bio-Rad) según instrucciones del fabricante. Se consideró como positivo un índice mayor a 0,5 tanto para muestras de suero como para LBA.

\section{Análisis y pruebas estadísticas}

Con los datos anteriores se calculó la sensibilidad, especificidad y likelihood ratio (LR) positivo y negativo para el LFD. Se determinó además la concordancia entre los resultados del LFD y GM calculándose el índice de Kappa. Para el análisis estadístico se utilizaron las pruebas de $\chi^{2}$ cuadrado y Fisher test según corresponda, mediante programa estadístico OpenEpi y GraphPad software.

Este proyecto fue aprobado por el Comité de Ética de la Facultad de Medicina de la Pontificia Universidad Católica de Chile (Número de protocolo 141203005, enero de 2015).

\section{Resultados}

Se analizó un total de 142 muestras (113 sueros y 29 LBA) de 98 pacientes cuya edad fue de $50 \pm 18$ años, con un predominio del sexo masculino (60,2\%). El 43,8\% de los pacientes presentaba como enfermedad de base una neoplasia hematológica siendo más frecuente la leucemia mieloide aguda $(31,6 \%)$. Los factores de riesgo para el desarrollo de AI fueron neutropenia profunda $(<100$ neutrófilos $/ \mathrm{mm}^{3}$ ) en $31,6 \%$ y uso de terapia inmunosupresora no corticoesteroidal en $27,5 \%$ (Tabla 1 ).

En el grupo de pacientes neutropénicos, el diagnóstico de EFI se realizó, en promedio, a los 10 días de iniciado el cuadro de neutropenia. De los pacientes incluidos, ocho tenían un cultivo positivo para Aspergillus, siendo

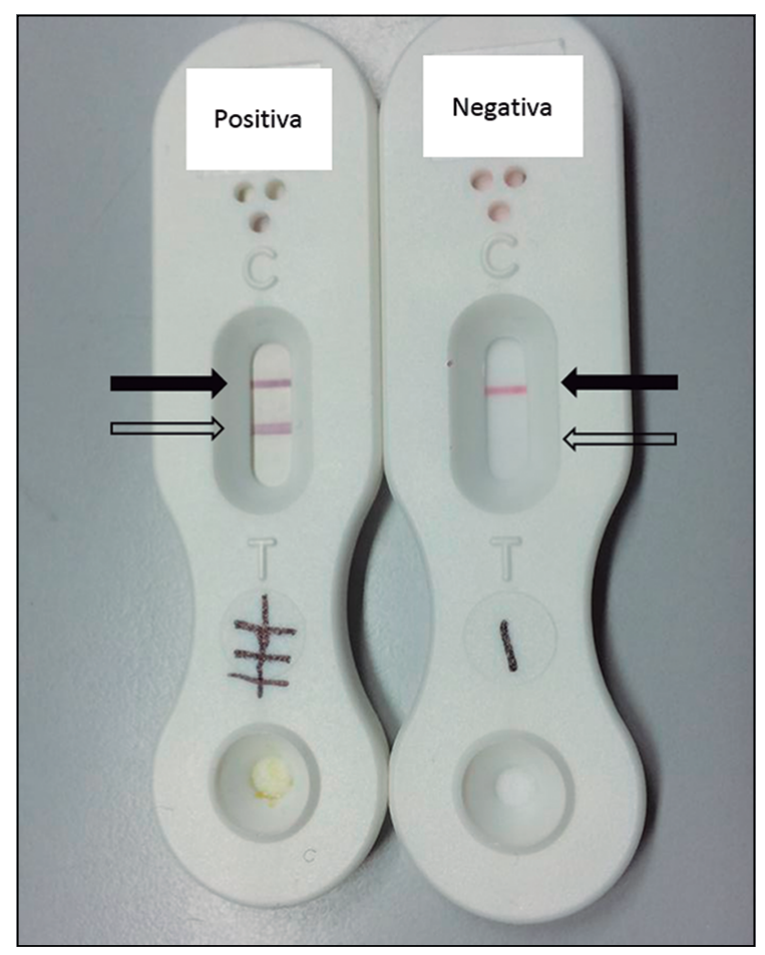

Figura 1. Lectura de resultado de LFD de dos muestras distintas, se pueden apreciar hasta dos bandas, la banda de control (identificado con flecha sólida negra) y luego una segunda banda (identificado con flecha sin relleno), cualquier intensidad se debe interpretar positiva. En la imagen, a izquierda resultado positivo y a derecha resultado negativo (ausencia de banda). 
Tabla 1. Características de 98 pacientes cuyas muestras de suero o lavado broncoalveolar habían sido utilizadas previamente para determinación de galactomamano por vigilancia, sospecha o seguimiento de aspergilosis invasora, que fueron sometidas a prueba de lateral flow device

\begin{tabular}{lc}
\hline Edad, años (promedio \pm DS) & $\mathbf{n}(\%)$ \\
\hline Sexo masculino & $50,9 \pm 18$ \\
\hline Enfermedad de base & $59(60,2)$ \\
Neoplasia hematológica & \\
Trasplante de precursores hematopoyéticos & $46(46,9)$ \\
Trasplante de órgano sólido & $16(16,3)$ \\
Tumor sólido & $6(6,1)$ \\
VIH/SIDA & $5(5,1)$ \\
Otro & $5(5,1)$ \\
\hline RAN $<500$ céls/mm ${ }^{3}$ & $20(20,4)$ \\
\hline RAN $<100$ céls/mm mm $^{3}$ & $42(42,9)$ \\
\hline Uso de corticosteroides & $31(31,6)$ \\
\hline Terapia inmunosupresora & $22(22,4)$ \\
\hline
\end{tabular}

RAN: recuento absoluto de neutrófilos.
Aspergillus sección Fumigati el más frecuentemente aislado, en cinco de ellos (62,5\%), Aspergillus sección Flavi en dos (25\%) y Aspergillus sección Terrei en un paciente $(12,5 \%)$.

Aplicando los criterios EORTC/MSG ${ }^{5}$, de las 142 muestras incluidas, ocho correspondían a AI probada $(5,6 \%), 59$ a AI probable (41,5\%), 18 a AI posible $(12,7 \%)$ y 57 a ausencia de AI (40,1\%).

Los resultados obtenidos por LFD se muestran en la Tabla 2. De las 142 muestras analizadas, LFD fue positivo en $88(61,9 \%)$ y negativo en $53(37,4 \%)$, no pudiendo interpretarse en uno $(0,7 \%)$.

Al analizar los resultados del LFD por tipo de muestra, observamos que, de las 113 muestras de suero analizadas, LFD fue positivo en $66(58,4 \%)$, negativo en $46(40,7 \%)$ no pudiendo interpretarse en una muestra $(0,8 \%)$. Por otra parte, al comparar los resultados de LFD entre pacientes neutropénicos versus no neutropénicos, observamos que en las muestras de suero LFD fue positivo en 48/77 $(62,3 \%)$ vs $18 / 36(50 \%) \mathrm{p}=0,049$, respectivamente.

Para el cálculo de sensibilidad, especificidad y LR se excluyeron las muestras de casos clasificados como AI posible y las muestras no interpretables. Los resultados por tipo de muestra se presentan en la Tabla 3. La sensibilidad y especificidad global fueron de 73,1 y $50 \%$, respectivamente. $\mathrm{Al}$ analizar según tipo de muestra

Tabla 2. Resultados de lateral flow device de las 142 muestras, 113 de suero y 29 de lavado bronco-alveolar, agrupadas según categoría diagnóstica de aspergilosis invasora

\begin{tabular}{lccccccc} 
Resultado LFD & \multicolumn{2}{c}{ Al Probada/Probable } & \multicolumn{2}{c}{ Al Posible } & \multicolumn{2}{c}{ Ausencia de Al } & \multicolumn{2}{c}{ Total } \\
& Suero & LBA & Suero & LBA & Suero & LBA & Suero y LBA \\
& $\mathbf{n}=\mathbf{5 5}(\%)$ & $\mathbf{n = 1 2 ( \% )}$ & $\mathbf{n = 1 4 ( \% )}$ & $\mathbf{n = 4 ( \% )}$ & $\mathbf{n = 4 4 ( \% )}$ & $\mathbf{n = 1 3 ( \% )}$ & $\mathbf{n = 1 4 2 ( \% )}$ \\
Positivo & $39(70,9)$ & $10(83,3)$ & $7(50)$ & $4(100)$ & $20(45,4)$ & $8(61,5)$ & $88(61,9)$ \\
Negativo & $16(29)$ & $2(16,7)$ & $7(50)$ & - & $23(52,3)$ & $5(38,5)$ & $53(37,3)$ \\
No interpretable & - & - & - & - & $1(2,3)$ & - & $1(0,7)$
\end{tabular}

Según criterios EORTC/MSG ${ }^{5}$. Al: aspergilosis invasora. LBA: lavado bronco-alveolar.

Tabla 3. Sensibilidad, especificidad y likelihood ratio (LR) de lateral flow device en 123 muestras de suero y lavado bronco-alveolar*

\begin{tabular}{lcccc}
\hline & $\begin{array}{c}\text { Sensibilidad } \\
\%(\text { IC 95\%) }\end{array}$ & $\begin{array}{c}\text { Especificidad } \\
\% \text { (IC 95\%) }\end{array}$ & LR (+) & LR (-) \\
Suero & $70,9(57,86-81,23)$ & $53,5(38,92-67,49)$ & $1,525(1,354-1,717)$ & $0,5439(0,4468-0,662)$ \\
LBA & $83,3(55,2-95,3)$ & $38,5(17,71-64,48)$ & $1,354(1,019-1,799)$ & $0,4333(0,08686-0,162)$ \\
Global & $73,1(61,48-82,28)$ & $50,0(37,33-62,67)$ & $1,463(1,344-1,592)$ & $0,5373(0,4493-0,426)$ \\
\hline
\end{tabular}

*Se excluyen 18 casos de aspergilosis posible y un caso con resultado lateral flow device no interpretable. 


\begin{tabular}{|c|c|c|}
\hline \multirow[b]{2}{*}{ LFD } & \multicolumn{2}{|c|}{ GM } \\
\hline & $\begin{array}{c}\text { Negativo } \\
\mathrm{n}=55\end{array}$ & $\begin{array}{l}\text { Positivo } \\
\mathrm{n}=87\end{array}$ \\
\hline $\begin{array}{l}\text { Negativo } \\
n=53\end{array}$ & 27 & 26 \\
\hline $\begin{array}{l}\text { Positivo } \\
\mathrm{n}=88\end{array}$ & 27 & 61 \\
\hline $\begin{array}{l}\text { No interpretable } \\
\mathrm{n}=1\end{array}$ & 1 & 0 \\
\hline
\end{tabular}

LFD: lateral flow device. GM: galactomanano. obtuvimos una sensibilidad y especificidad de $70,9 \%$ y $53,5 \%$ para muestras de suero mientras que para LBA fueron de $83,3 \%$ y $38,5 \%$.

Por otro lado, al analizar los resultados según tipo de paciente observamos una sensibilidad y especificidad de $70,7 \%$ y $45,8 \%$ para muestras de suero de pacientes neutropénicos y en cambio de $73,7 \%$ y $54,2 \%$ para muestras de pacientes no neutropénicos.

Al comparar los resultados de LFD con GM del total de muestras, obtuvimos una concordancia general entre ambos métodos de 62,4\% (54,1\%-69,9\%). El índice de concordancia Kappa calculado fue de 0,202 (0,036820,3669), considerado como un acuerdo mediano (Tabla 4). Al realizar esta comparación sólo en el grupo de pacientes neutropénicos se observó una concordancia de $66,2 \%$ (42,2\%-69,3\%) con un índice de Kappa de 0,28 (0,079$0,496)$, mientras que en las muestras correspondientes a pacientes no neutropénicos la concordancia fue de 56,2\% (42,2\%-69,3\%) con un índice Kappa de 0,066 (-0,2-0,34), es decir la concordancia fue igual al azar.

\section{Discusión}

En este estudio se evaluó el rendimiento de LFD en pacientes adultos, tanto para muestras de suero como LBA, que habían sido evaluados por vigilancia, sospecha o seguimiento de AI, constatando una sensibilidad de $73,1 \%$, especificidad de $50 \%$ y una concordancia mediana con GM, evidenciando un leve mejor desempeño en pacientes neutropénicos que no-neutropénicos.

Diversos estudios publicados en la literatura han evaluado el desempeño de esta prueba con resultados generales satisfactorios. En un estudio del Reino Unido, que incluyó 103 adultos con patología hemato-oncológica en alto riesgo de $\mathrm{AI}$, con ocho casos de AI probada, 14 probable, 22 posible y $59 \sin$ AI, se describe una sensibilidad de $81 \%$ con una especificidad de $98 \%{ }^{10}$. Por otro lado, un estudio experimental en modelos animales de AI que compara las pruebas LFD con GMN y $\beta$-D-glucano en suero, demuestra la mayor precocidad de LFD en evidenciar $\mathrm{AI}^{11}$. Por su parte, Hoenigl y cols., publicaron resultados de LFD para muestras de LBA en pacientes con cáncer hematológico y receptores de órganos sólidos reportando sensibilidades de 80 a $100 \%$, con especificidades de 81 a $98 \%$ y valores predictores positivo y negativo de 71 y $100 \%$, respectivamente ${ }^{12}$.

Si bien en nuestro estudio observamos un desempeño global comparable a lo publicado en términos de sensibilidad, destaca la baja especificidad observada, en especial para muestras de LBA. Ahora bien, en un estudio multicéntrico publicado por Eigl y cols., en que se evaluó el rendimiento diagnóstico de LFD en 149 muestras de LBA de pacientes críticos con patología pulmonar o cardiaca y pacientes hemato-oncológicos en cuatro centros en Austria y Alemania, se describe una especificidad de $54 \%$, que resulta más cercana a la observada en nuestro estudio $^{13 .}$

Respecto a la concordancia mediana a pobre entre GM y LFD presentada en los resultados de nuestra serie, contrastan con un estudio chileno publicado por Álvarez en que se evaluó la concordancia entre GM y LFD en 29 muestras de suero y LBA, que incluía solo tres muestras con GM negativo, constatando una concordancia de resultado de ambas pruebas en $100 \%$ de los $\operatorname{casos}^{14}$. Esta diferencia respecto a la menor concordancia observada en nuestro estudio, podría estar explicada por el bajo número de muestras con resultado de GM negativo, grupo de muestras que presentaron el mayor número de discordancias en nuestra serie. Al revisar las discordancias entre LFD y GM de nuestros casos, en los 27 en que se obtuvo un resultado de LFD positivos con GM negativo, el promedio de GM obtenido fue de $0,1(0,01$ $0,43)$, muy por debajo del punto de corte. En cinco de estos casos se tenía medición de $\beta$-D-glucano, habiendo resultado negativo en cuatro de ellos. El único resultado positivo presentaba una concentración de $326 \mathrm{pg} / \mathrm{mL}$ y correspondía a un paciente con diagnóstico de infección fúngica por Fusarium sp. por lo que el resultado positivo de LFD podría corresponder a una reacción cruzada con este hongo. Por otro lado, en el caso de las muestras con resultados de LFD negativo con GM positivo, el promedio del GM fue de 1,6 (0,5-9,9). A pesar de estas situaciones, dada la complejidad del diagnóstico de AI, consideramos que la concordancia entre ambos resultados no sería necesariamente el parámetro único o más relevante para definir el desempeño de este test.

Factores que pudiesen influir en el desempeño de LFD han sido planteados en algunos trabajos. Uno de los factores es la subjetividad de la interpretación del 
resultado; según el fabricante, toda línea debe ser considerada positiva. Debemos aclarar que, durante nuestra experiencia, el test no contaba con controles por lo que no se pudo determinar la presencia de marcas muy tenues o inespecíficas. En un estudio de Held y cols., se utilizaron muestras séricas de sujetos sanos para poder establecer una línea de positividad, la cual fue cotejada con sujetos receptores de trasplante hematopoyético que desarrollaron EFI en el período de seguimiento del estudio antes y después del trasplante ${ }^{15}$. Este paso podría disminuir la interpretación de resultados inespecíficos como positivos, pero introduce subjetividad en el análisis de los resultados. Otro factor, similar a lo descrito para GM, es que el uso de antifúngicos podría disminuir la sensibilidad de la prueba ${ }^{16}$ aumentando el número de resultados falsamente negativos. Sin embargo, faltan mayores estudios que evalúen este aspecto o si, eventualmente, LDF tendría alguna utilidad en el seguimiento de la respuesta terapéutica de los pacientes con EFI. Otra consideración a tener presente para explicar el menor desempeño al esperado es haber procesado muestras congeladas por más de seis meses; dos estudios han utilizado muestras conservadas en similares condiciones hasta por seis años ${ }^{16,17} \sin$ afectar el desempeño observado.

Este año se ha publicado interesantes resultados de un estudio austriaco con muestras de LBA de 23 pacientes con cáncer hematológico y cinco pacientes críticos en que se evaluó una nueva versión de LFD (OLM Diagnostics ${ }^{\circledR}$ ) y se comparó con el prototipo utilizado en nuestro estudio, observando una mayor sensibilidad y especificidad: 71 y $100 \%$ con la nueva versión vs. 64 y $64 \%$ con el prototipo previo $^{18}$

En la medida que el test se encuentre disponible para uso rutinario, se deberá establecer su incorporación en los protocolos diagnósticos de AI. Considerando sus características en cuanto al breve tiempo de obtención de resultados, baja demanda de recursos y eventuales bajos costos, podría plantearse su uso como parte de una vigilancia más intensiva que la que es posible realizar con GM en la actualidad. Este tipo de estrategia deberá ser evaluada en un estudio diseñado para este fin.

Esta experiencia nos permite señalar que LFD es un test de fácil realización no requiriendo de un laboratorio de gran complejidad. No obstante, el procesamiento de las muestras de suero necesita de un paso de centrifugación y calentamiento, lo cual limita su uso como prueba al lado de la cama del paciente ("point of care testing"). Otro aspecto a señalar es la importancia de mejorar la estandarización de la lectura del resultado para aquellos que presentan dificultad en la interpretación, a fin de eliminar la subjetividad del evaluador. La existencia de una nueva versión de LFD plantea el desafío de conocer si el rendimiento en muestras de suero o de otro tipo y en diferentes grupos de pacientes se logra replicar el beneficio observado en $\mathrm{LBA}^{18}$, para lo que se requerirán nuevos estudios que respondan estas preguntas.

En conclusión, los resultados obtenidos en este trabajo sugieren una adecuada sensibilidad del test lo que podría otorgarle un valor como prueba de cribado para AI en pacientes de alto riesgo. Sin embargo, dada la baja especificidad, de considerarse su uso en la práctica clínica, todo resultado positivo deberá ser confirmado con otras pruebas diagnósticas.

Previo a su incorporación, se hace necesario contar con más información proveniente de nuevos estudios que permitan establecer la validación de esta prueba en un rol de cribado, definir su rendimiento en estrategia de vigilancia o para el seguimiento de casos de AI en terapia antifúngica, establecer su rol en diferentes grupos de pacientes y conocer su aporte en la potenciación del proceso diagnóstico con el uso combinado de exámenes serológicos y de biología molecular, entre otros.

\section{Resumen}

Introducción: El diagnóstico de aspergilosis invasora (AI) se realiza mediante criterios clínicos y microbiológicos los que incluyen marcadores séricos. Recientemente, el test inmunocromatográfico Aspergillus lateral flow device (LFD), ha sido evaluado como método para diagnóstico de AI. Objetivo: Evaluar el desempeño de este test para el diagnóstico de AI. Material y Método: Estudio transversal en que se evaluaron muestras de suero y lavado bronco-alveolar (LBA) procesadas para galactomanano provenientes de pacientes adultos con sospecha de AI, atendidos en el Hospital Clínico de Red de Salud UCCHRISTUS. Resultados: Se procesó un total de 142 muestras de 98 pacientes, correspondientes a AI probada $5,6 \%$, AI probable $41,5 \%$, AI posible $12,7 \%$ y ausencia de AI 40,1\%. Al confrontar los resultados con las categorías diagnósticas según criterios EORTC/MSG se obtuvo una sensibilidad y especificidad de LFD para diagnóstico de AI de 70,9 y 53,5\% para muestras de suero y 83,3 y $38,5 \%$ para muestras de LBA. La concordancia entre galactomanano y LFD fue de $62,4 \%(54,1-69,9)$ con un índice Kappa de 0,202 (0,03682-0,3669). Conclusiones: Aspergillus LFD presentó una adecuada sensibilidad; sin embargo, la especificidad fue baja por lo que un resultado positivo requiere ser confirmado. 


\section{Referencias bibliográficas}

1.- Cuenca-Estrella M, Bernal-Martínez L, Buitrago M J, Castelli M V, Gómez López A, Zaragoza $\mathrm{O}$, et al. Update on the epidemiology and diagnosis of invasive fungal infection. Int $\mathrm{J}$ Antimicrob Agents 2008; 32: S143-S147. doi: 10.1016/S0924-8579(08)70016-5.

2.- Azie N, Neofytos D, Pfaller M, Meier-Kriesche HU, Quan S P, Horn D. The PATH (Prospective Antifungal Therapy) Alliance $\AA$ registry and invasive fungal infections: update 2012. Diagn Microbiol Infect Dis 2012; 73: 293-300. doi: 10.1016/j.diagmicrobio.2012.06.012.

3.- Rabagliati R, Fuentes G, Orellana E. Oporto J, Domínguez I, Benítez R, et al. Etiología de episodios de neutropenia febril en pacientes adultos con cáncer hematológico y de órganos sólidos en el Hospital Clínico Universidad Católica, Santiago-Chile. Rev Chilena Infectol 2009; 26: 212-9. http://dx.doi.org/10.4067/ S0716-10182009000200001.

4.- Rabagliati R, Bertin P, Cerón I, Rojas H, Domínguez I, Vera A, et al. Epidemiología de neutropenia febril en pacientes adultos con leucemia aguda y linfoma. Estudio de cohorte de hospital público y privado de Santiago, Chile. Rev Chilena Infectol 2014; 31: 721-8 doi: 10.4067/S0716-10182014000600013.

5.- De Pauw B, Walsh T J, Donnelly J P, Stevens D A, Edwards J E, Calandra T, et al. Revised definitions of invasive fungal disease from the European Organization for Research and Treatment of Cancer/Invasive Fungal Infections Cooperative Group and the National Institute of Allergy and Infectious Diseases Mycoses Study Group (EORTC/MSG) Consensus Group. Clin Infect Dis 2008; 46: 1813-21. doi: $10.1086 / 588660$.

6.- White P L, Bretagne S, Klingspor L, Melchers W J, McCulloch E, Schulz B, et al. On behalf of the European Aspergillus PCR initiative. Aspergillus PCR: one step closer to standardization. J Clin Microbiol 2010; 48: 1231-40. doi: 10.1128/JCM.01767-09.

7.- Marchetti O, Lamoth F, Mikulska M, Viscoli C, Verweij P, Bretagne S, and the European Conference on Infections in Leukemia (ECIL) Laboratory Working Groups. ECIL recommendations for the use of biological markers for the diagnosis of invasive fungal diseases in leukemic patients and hematopoietic SCT recipients. Bone Marrow Transplant 2012; 47: 846-54. doi: 10.1038/bmt.2011.178.

8.- Koo S, Bryar J M, Badem, L R, Marty F M. Prognostic features of galactomannan antigenemia in galactomannan-positive invasive aspergillosis. J Clin Microbiol 2010; 48: 1255-60. doi: 10.1128/JCM.02281-09.

9.- Thornton C R. Development of an immunochromatographic lateral-flow device for rapid serodiagnosis of invasive aspergillosis. Clin Vaccine Immunol 2008; 15: 1095-105. doi: 10.1128/CVI.00068-08.

10.- White P L, Barr C, Thornton C, Barnes R A. Evaluation of real-time PCR, galactomannan enzyme-linked immunosorbent assay (ELISA), and a novel lateral-flow device for diagnosis of invasive aspergillosis. J Clin Microbiol 2013; 51: 1510-16. doi: 10.1128/JCM.03189-12.

11.- Wiederhold N P, Thornton C R, Najvar K, Kirkpatrick W R, Bocanegra R, Patterson F T. Comparison of lateral flow technology and galactomannan and (1-3)-b-d-glucan assays for detection of invasive pulmonary aspergillosis. Clin Vaccine Immunol 2009; 16: 1844-6. doi:10.1128/CVI.00268-09.

12.- Hoenigl M, Koidl C, Duettmann W, Seeber K, Wagner J, Buzina W, et al. Bronchoalveolar lavage lateral-flow device test for invasive pulmonary aspergillosis diagnosis in haematological malignancy and solid organ transplant patients. J Infect 2012; 65: 588-91 doi: 10.1016/j.jinf.2012.10.003.

13.- Eigl S, Prattes P, Lackner M, Willinger B, Spiess B, Reinwald M, et al. Multicenter evaluation of a lateral-flow device test for diagnosing invasive pulmonary aspergillosis in ICU patients. Critical Care 2015; 19: 178. https://doi.org/10.1186/s13054-015-0905-x.

14.- Álvarez E. Utilidad de la prueba AspergillusLFD para el diagnóstico de aspergilosis: primera experiencia en Chile. Rev Chilena Infectol 2015; 32: 117-19. http://dx.doi. org/10.4067/S0716-10182015000200017.

15.- Held J, Schmidt T, Thornton C R, Kotter C, Bertz H. Comparison of a novel Aspergillus lateral-flow device and the Platelia galactomannan assay for the diagnosis of invasive aspergillosis following haematopoietic stem cell transplantation. Infection 2013; 41: 1163-69. doi: 10.1007/ s15010-013-0472-5.

16.- Wiederhold N, Najvar L, Bocanegra R, Kirkpatrick W R, Patterson T F, Thornton C R. Interlaboratory and interstudy reproducibility of a novel lateral-flow device and influence of antifungal therapy on detection of invasive pulmonary aspergillosis. J Clin Microbiol 2013; 51: 459-65. doi: 10.1128/JCM.02142-12.

17.- Johnson G, Sarker S H, Nannini F, Ferrini A, Taylor E, Lass-Florl C, et al. Aspergillus specific lateral flow device and real time pcr testing of bronchoalveolar lavage fluid: a combination biomarker approach for clinical diagnosis of invasive pulmonary aspergillosis. $\mathrm{J}$ Clin Microbiol 2015; 53: 2103-8. doi: 10.1128/ JCM.00110-15.

18.- Hoenigl M, Eigl S, Heldt S, Duettmann W, Thornton C, Prattes J. Clinical evaluation of the newly formatted lateral-flow device for invasive pulmonary aspergillosis. Mycoses. 2018; 61: 40-43. doi: 10.1111/myc.12704. 